\title{
COMPREHENSIVE STUdy OF ACOUSTIC CHANNEL MODELS FOR UNDERWATER WIRELESS COMMUNICATION NETWORKS
}

\author{
S Anandalatchoumy ${ }^{1}$ and G Sivaradje ${ }^{2}$ \\ Department of Electronics and Communication Engineering, Pondicherry Engineering \\ College, Pondicherry, India.
}

\begin{abstract}
In underwater acoustic communication, shallow water and deep water are two different mediums which exhibit many challenges to deal with due to the time varying multipath and Doppler Effect in the former case and multipath propagation in the latter case. In this paper, the characteristics of the acoustic propagation are described in detail and channel models based on the various propagation phenomena in shallow water channel and deep water channel as well are presented and the transmission losses incurred in each model are thoroughly investigated. Signal to noise ratio (SNR) at the receiver is thoroughly analyzed. Numerical results obtained through analytical simulations carried out in MATLAB bring to light the important issues to be considered so as to develop suitable communication protocols for Underwater Wireless Communication Networks (UWCNs) to provide effective and reliable communication.
\end{abstract}

\section{KEYWORDS}

Underwater Acoustic Communication, Transmission Loss, Shallow Water, Deep Water.

\section{INTRODUCTION}

About $70 \%$ of the earth is surrounded by water in the form of oceans. Wireless signal transmission underwater will enable many different applications to be performed in highly dynamic and harsh environment of underwater medium which would benefit the mankind to enhance their life [1]. So, tremendous success of the application of wireless sensor technology in the development of terrestrial wireless sensor networks has motivated the scientists and researchers to apply the same technology to explore the less unexplored ocean to benefit mankind from the ocean natural resources and to rescue them from natural disasters like Tsunamis, earthquakes and so on. This has paved the way for them to develop Underwater Wireless Communication Networks (UWCNs).

Basically, UWCNs are formed by group of sensors and/or autonomous underwater vehicles deployed underwater and networked through wireless links by acoustic signals to perform collaborative monitoring tasks over a given area [1]. These networks are also referred to as Underwater Wireless Sensor Networks. Underwater acoustic communication channel exhibits challenges in many aspects which are quite different from the terrestrial radio communication channel due to the acoustic propagation characteristics such as transmission loss, multipath propagation and multipath fading, Doppler spreading, ambient noise, time-dependent channel variations and variable delay. This makes underwater wireless communication more challenging. Moreover, higher frequencies experience more absorption loss resulting in the limit of usable bandwidth. This necessitates studying the propagation characteristics of the acoustic channel in 
International Journal on Cybernetics \& Informatics (IJCI) Vol. 4, No. 2, April 2015

order to facilitate the efficient deployment of sensor nodes and development of higher layer communication protocols for UWCNs to ensure effective and reliable communication.

In this paper, a comprehensive study of the acoustic propagation characteristics and acoustic channel models are provided with detailed discussions on the design issues and research challenges for effective communication in underwater environment. This paper is organized into 6 sections as follows: Section 2 outlines some of the existing literature related to the acoustic channel models, Section 3 discusses the fundamental aspects of ocean acoustics and introduces the main aspects of the propagation characteristics of the underwater acoustic channel, Section 4 discusses the transmission losses incurred in shallow and deep water channel and ambient noise in the ocean, Section 5 analyzes the signal to noise ratio at the receiver and Section 6 concludes this paper highlighting the important considerations for the design of UWCNs for effective communication.

\section{RELATED WORK}

The model proposed in [2] is a very simple underwater acoustic channel model to generate a reliable prediction of the transmission loss that increases in proportion to the acoustic frequency.Underwater channel models proposed in the literature are simple. The transmission loss is computed by considering only the effects of spreading effect and absorption effect [3]. The propagation loss formula combined with a stochastic fading component calculated from two Gaussian variables is used to compute transmission loss [4]. However, these models do not do not consider several effects such as multipath, fading, shadow zones, which exist in underwater environments.

Authors in [5] proposed a Rayleigh fading channel model for shallow water but there is still no single model available as accepted by the entire research community which is applicable for shallow waters [6]. In [7], a simple stochastic channel model is proposed and no experimental results are presented. Moreover, the model does not include acoustic propagation physics, e.g., spreading and absorption.. An underwater acoustic channel based on Rice fading model is introduced in [8] where there can be several propagation paths named Eigen paths and the Poisson distribution is used to represent the number of Eigen paths reaching a receiver. Themodelsproposed in [9] are simple deep water acoustic models. These models include acoustic propagation physics such as spreading and absorption and captures transmission losses due to sea surface and sea bottom. They do not however consider the real time effect of different propagation phenomena in the deep underwater such as surface duct, convergence zones, deep sound channel and reliable acoustic paths.

This paper presents a comprehensive study of underwater acoustic channel modelsbased on the characteristics of sound propagation due to various modes for shallow and deep water. Shallow water challenges such as time-varying multipath due to multiple reflections from the sea surface and the sea bottom are focused and the effects of all existing different propagation phenomena such as surface reflection, surface duct, bottom bounce, convergence zone, deep sound channel, reliable acoustic paths in the deep water are analyzed. The research challenges for deployment, localization and routing schemes for UWCNs are also pointed out based on numerical results. 
International Journal on Cybernetics \& Informatics (IJCI) Vol. 4, No. 2, April 2015

\section{Fundamentals of OCEAN ACOUSTICS}

The ocean is an extremely complicated and dynamic acoustic medium with the characteristic feature of inhomogeneous nature. Regular and Random are two kinds of heterogeneities observed in the ocean which cause fluctuations in the sound field. Hence, the speed of sound varies with depth, temperature, salinity, location, time of the day and season. Basically, ocean acoustic channel is divided into two channels: Shallow water channel and Deep water channel. Shallow water refers to water column with depth $100 \mathrm{~m}$ and below whereas deep water refers to water column with depth above $100 \mathrm{~m}$.

\subsection{Sound Propagation in the Ocean}

The study of sound propagation in the ocean is vital to the understanding of wireless signal transmission underwater through acoustic channel. Sound propagation in the ocean is influenced by the physical and chemical properties of the seawater and by the geometry of the channel itself [10]. Sound propagates in the ocean with variable sound velocity. Sound velocity varies with variations in temperature, salinity and depth. Variations of the sound velocity are relatively small. However, even small changes cansignificantly affect the propagation of sound in the ocean.

An empirical formulafor estimating sound velocity as a function of temperature, salinity and depth is given by Mackenzie and found to be more appropriate [11]:

$$
\begin{aligned}
& c=1449+4.591 T-5.304 \times 10^{-2} T^{2}+2.374 \times 10^{-4} T^{3}+1.34(S-35)+1.63 \times 10^{-2} D+1.675 \times 10^{-7} D^{2} \\
& +1.025 \times 10^{-2} T(S-35)-7.139 \times 10^{-3} T D^{3}
\end{aligned}
$$

where, ' $c$ ' is the sound speed expressed in $\mathrm{m} / \mathrm{s}$, ' $\mathrm{T}$ ' is temperature expressed in ${ }^{\circ} \mathrm{C}$, salinity ' $\mathrm{S}$ ' is salinity expressed in parts per thousand (ppt) and ' $\mathrm{D}$ ' is depth expressed in meters. Eqn. (1) is valid for $0^{\circ}<T \leq 30^{\circ} \mathrm{C} ; \quad 30 \mathrm{ppt} \leq S \leq 40 \mathrm{ppt} ; 0 \leq \mathrm{D}<=8000 \mathrm{~m}$. Figure 1illustrates the Sound velocity profile. The impact of temperature, salinity and depth upon the sound velocity in the ocean can be observed in three layers of regions with different depth ranges. First layer region is from the surface to a depth of $200 \mathrm{~m}$ from the surface which contains surface layer ( 0 to $100 \mathrm{~m}$ ) delimited by red line in the figure and seasonal thermocline layer (100m to $200 \mathrm{~m})$ delimited by green line. Second layer region is from $200 \mathrm{~m}$ depth to $1000 \mathrm{~m}$ depth which contains main thermocline layer delimited by magenta line. In this layer, sound velocity decreases primarily due to rapidly decreasing temperature. Third layer is from $1000 \mathrm{~m}$ depth to ocean bottom which contains deep isothermal layer. In this layer, temperature becomes almost constant of about $2^{\circ}$ celsius and sound speed increases linearly only due to increase in depth. Sound velocity is highly variable depending upon the depth, temperature, salinity, season and time of the day. Its value usually ranges from $1450 \mathrm{~m} / \mathrm{s}$ to $1560 \mathrm{~m} / \mathrm{s}$ [10].

\subsection{Propagation Loss of Sound}

An underwater acoustic signal experiences attenuation due to spreading, scattering and absorption [3].

\subsubsection{Spreading Loss}


Spreading loss is a measure of signal weakening due to the effect of geometrical spreading when a sound wave propagates away from the source. . Spherical spreading and Cylindrical spreading are two kinds of spreading in underwater acoustics: The spreading loss is calculated using the formula given below [2]:

$$
P L_{\text {spreading }}=k 10 \log (r)
$$

where, $P L_{\text {spreading }}$ is the path loss expressed in $\mathrm{dB}, \mathrm{r}$ is the transmission range in meters and $\mathrm{k}$ is the spreading factor ( $\mathrm{k}=1$ for cylindrical spreading and $\mathrm{k}=2$ for spherical spreading).

\subsubsection{Absorption Loss}

The absorption loss represents the energy loss of sound due to the transformation of energy in the form of heat due to the chemical properties of viscous friction and ionic relaxation in the ocean and this loss is range-dependent and computed as follows:

$$
P L_{\text {absorption }}=\alpha r \times 10^{-3}
$$

where, $P L_{\text {absorption }}$ is the path loss expressed in $\mathrm{dB}, \mathrm{r}$ is transmission range in meters and $\alpha$ is the absorption coefficient in $\mathrm{dB} / \mathrm{km}$.

\section{AbsorptionCoefficient}

An empirical formula for absorption coefficient has been proposed which varies with frequency, pressure (depth) and temperature and is valid for frequency range of $100 \mathrm{~Hz}$ to $1 \mathrm{MHz}[12,13]$. It is expressed in $\mathrm{dB} / \mathrm{Km}$ and given as follows:

$$
\alpha=\underbrace{\frac{\mathrm{A}_{1} \mathrm{P}_{1} \mathrm{f}_{1} \mathrm{f}^{2}}{f_{1}{ }^{2}+f^{2}}}_{(1)}+\underbrace{\frac{\mathrm{A}_{2} \mathrm{P}_{2} \mathrm{f}_{2} \mathrm{f}^{2}}{f_{2}{ }^{2}+f^{2}}}_{(2)}+\underbrace{\mathrm{A}_{3} \mathrm{P}_{3} \mathrm{f}^{2}}_{(3)}[\mathrm{dB} / \mathrm{km}]
$$

The first term in Eqn. (4) corresponds to the contribution of boric acid, $\mathrm{BOH}_{3}$, the second term corresponds to the contribution of magnesium sulphate, $\mathrm{MgSO}_{4}$ and the third term corresponds to the contribution of pure water, $\mathrm{H}_{2} \mathrm{O}$ to the absorption loss of the acoustic signal in sea water. The effects of temperature, the ocean depth (pressure) and the relaxation frequencies of boric acid and magnesium sulphatemoleculesare represented by ' $A$ ' coefficients, ' $P$ ' coefficients, and $f_{1}, f_{2}$ respectively. 
International Journal on Cybernetics \& Informatics (IJCI) Vol. 4, No. 2, April 2015
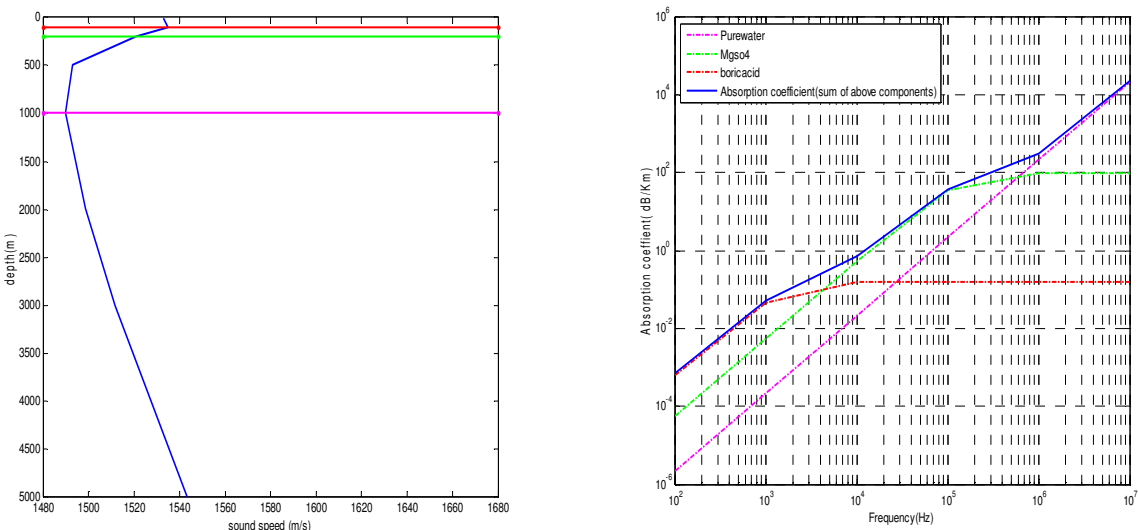

Figure 1.Sound velocity profile Figure 2. Absorption Coefficient

Figure 2 represents the influence of various components and parameters of attenuation. It can be clearly noted that boric acid is dominant factor in contributing to attenuation coefficient for frequencies in the range below $1 \mathrm{kHz}$, for frequency range $1 \mathrm{kHz}$ to $100 \mathrm{kHz}$ magnesium sulphate and for frequency above $100 \mathrm{kHz}$ pure water contributes more to attenuation coefficient. It can also be observed that attenuation increases very rapidly with frequency and that the orders of magnitude are highly variable. For frequencies of $1 \mathrm{kHz}$ and below, attenuation is below a few hundredths of $\mathrm{dB} / \mathrm{km}$ and is therefore not a limiting factor. At $10 \mathrm{kHz}$, attenuation is about 1 $\mathrm{dB} / \mathrm{km}$ precluding ranges of more than tens of kilometers. At $100 \mathrm{kHz}$, attenuation reaches several tens of $\mathrm{dB} / \mathrm{km}$ and the practical range cannot exceed $1 \mathrm{~km}$.

\section{TRANSMISSION LOSS}

Transmission loss (TL) is defined as the accumulated decrease in acoustic intensity when an acoustic pressure wave propagates outwards from a source. The magnitude is estimated by the sum of the magnitudes of geometrical spreading loss and absorptionloss. The TL value can be useful in determining the arriving signal strength of a data stream and even the minimum required signal strength necessary to successfully complete a transmission within an underwater wireless acoustic network.

\subsection{Transmission Loss in Shallow Water (Direct Path Model)}

Acoustic signals in shallow water propagate within a cylinder bounded by the surface and the sea floor resulting in cylindrical spreading. The transmission loss caused by cylindrical spreading and absorption can be expressed as follows [2]:

$$
T L=10 \log r+\alpha r \times 10^{-3}
$$

where, TL is the transmission loss expressed in $\mathrm{dB}, \alpha$ is the absorption coefficient expressed in $\mathrm{dB} / \mathrm{km}$ and $\mathrm{r}$ is the transmission range in meters. 
International Journal on Cybernetics \& Informatics (IJCI) Vol. 4, No. 2, April 2015

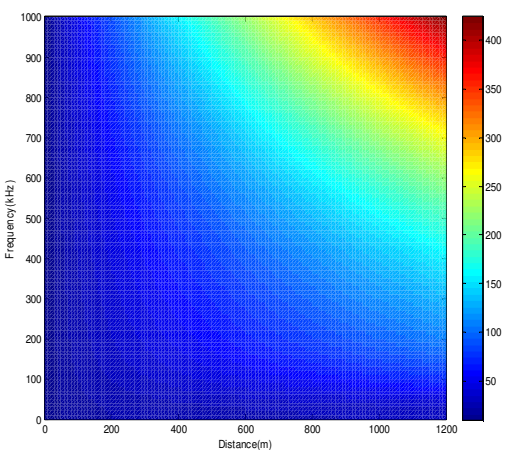

(a)

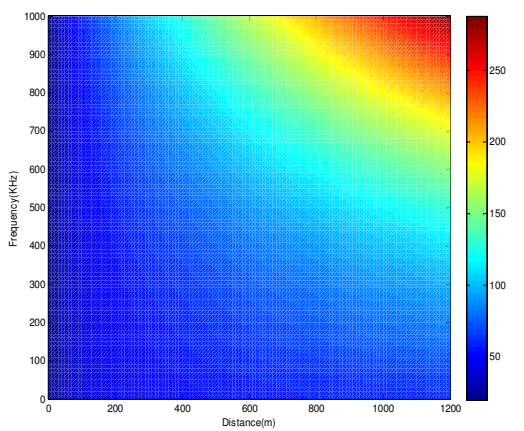

(b)

Figure 3.TL as a function of distance and frequency

(a) Direct path modelfor shallow water (b) Direct path model for deep water

Figure 3 (a) represents the total transmission loss in shallow water as a function of distance for different frequencies. It can be easily observed from the figure that inferred that transmission to larger distances requires low frequencies and at low frequencies, cylindrical spreading is the most important factor affecting TL because it is not frequency dependent.

\subsection{Transmission Loss in Deep Water (Direct Path Model)}

Deep water is considered to be a homogeneous unbounded medium and the transmission loss is caused due to spherical spreading and absorption. The transmission loss caused by spherical spreading and absorption can be expressed as follows [2]:

$$
T L=20 \log r+\alpha r \times 10^{-3}
$$

where TL is the transmission loss expressed in $\mathrm{dB}, \alpha$ is the absorption coefficient expressed in $\mathrm{dB} / \mathrm{Km}$ and $\mathrm{r}$ is the transmission range in meters.

Figure 3 (b) represents the total transmission loss in deep water as a function of distance for different frequencies. It can be well understood from the figure that transmission to larger distances requires low frequencies and at low frequencies, spherical spreading is the most important factor affecting TL because it is not frequency-dependent. The TL values in deep water are larger than in shallow water for low frequencies or low transmission distances because the spreading term dominates. TL in deep water is lower than TL in shallow water for large distances and higher frequencies since the absorption losses are the major cause of TL and they diminish with depth. Hence, it is clearly inferred that effective communication is possible when transceivers are located in deep water.

\subsection{Transmission Loss in Shallow Water (Multipath Model)}

Sound propagates to a distance in shallow water by repeated reflections from the surface and bottom resulting in multipath propagation [14]. Transmission loss calculations were completed using frequency dependent acoustic algorithms based on the semi-empirical step model of H.W. Marsh and M. Schulkin. The following equation is used when $r$, the horizontal separation distance 
International Journal on Cybernetics \& Informatics (IJCI) Vol. 4, No. 2, April 2015

between sound source and receiver is up to 1 times $\mathrm{H}$, which for the purposes of this analysis was conservatively defined as the average water depth of the acoustic study area [15]:

$T L=20 \log r+\alpha r+60-k_{L}(7)$

where,

$\mathrm{r}=$ horizontal separation distance between sound source and receiver (kilometers)

$\mathrm{H}=$ skip distance $($ in $\mathrm{km}$ ), defined as the maximum transition range at which rays make contact with either the surface or bottom and calculated as:

$H=\sqrt{1 / 3(d+z)}$

where,

$\mathrm{d}=$ mixed layer depth $(\mathrm{m}), \mathrm{z}$ - water depth $(\mathrm{m}), \alpha=$ shallow water absorption coefficient $\left(\mathrm{dB} /\right.$ kilometer) and $\mathrm{k}_{\mathrm{L}}=$ near-field anomaly. The intermediate (or transition zone) is defined for $\mathrm{H} \leq \mathrm{r} \leq 8 \mathrm{H}$ where modified cylindrical spreading occurs accompanied by mode stripping effects. The transmission loss equation representing this intermediate range is given as follows [15]:

$T L=15 \log r+\alpha r+\alpha_{T}(r / H-1)+5 \log H+60-k_{L}(9)$

where, $\alpha_{\mathrm{T}}=$ shallow water attenuation coefficient.

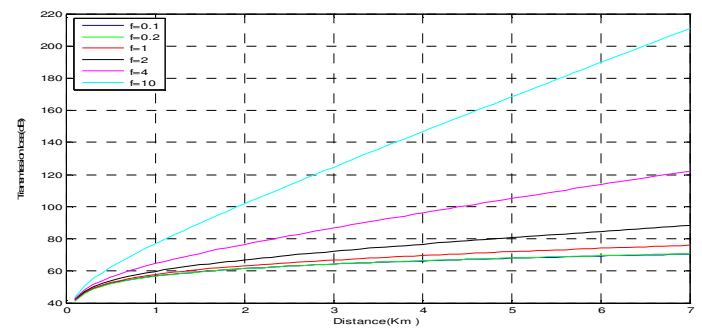

Figure 4. Transmission loss for various frequencies(Multipath model for shallow water)

Long range TL occurs where $r>8 \mathrm{H}$. Due to the boundaries of the sea surface and sea floor, sound energy is not able to propagate uniformly in all directions from a source indefinitely; therefore, long range TL is represented as cylindrical spreading, limited by the channel boundaries. Cylindrical spreading propagation is applied using the equation given below [15]:

$T L=10 \log r+\alpha r+\alpha_{T}(r / H-1)+10 \log H+60-k_{L}(10)$

where, $\alpha \mathrm{T}=$ shallow water attenuation coefficient $(\mathrm{dB})$.

The near-field anomaly $\left(\mathrm{k}_{\mathrm{L}}\right)$ and shallow water attenuation coefficient $\left(\alpha_{\mathrm{T}}\right)$ are functions of frequency, sea state, and bottom composition and their values are found in [2]. The anomaly term is related to the reverberant sound field developed near the source by surface and bottom reflected sound energy resulting in an apparent increase in source levels. The shallow water attenuation 
International Journal on Cybernetics \& Informatics (IJCI) Vol. 4, No. 2, April 2015

coefficient is an empirically determined factor related to sound scattering and other losses at water column boundaries.

Transmission loss in shallow water (multipath) with a bottom composition of sand and a sea state 1 is shown in Figure 4. It is clearly noted that TL increases with increasing frequency and distance. When compared with single path model, multipath model results in higher transmission loss due to reflections.

\subsection{Transmission Loss in Deep Water (Multipath Model)}

Sound propagates in deep water to a distance due to six propagation phenomena as detailed below resulting in multipath propagation. TL calculations for each category are derived at each different propagation path.

\subsubsection{Surface Reflection}

The loss on surface reflection as a function of the angle of incidence to the horizontal can be calculated using the Beckmann-Spizzichino model as follows [16]:

$T L_{S R}=10 \log \left[\frac{1+\left(f / f_{1}^{2}\right)}{1+\left(f / f_{2}^{2}\right)}\right]-(1+(90-w) / 60)(\theta / 30)^{2}$

where, $f_{1}=\sqrt{10} f_{2}, f_{2}=378 w^{-2}$, w-wind

$\operatorname{speed}(\mathrm{m} / \mathrm{s})$.

The total transmission loss as a result of surface reflection for near-surface source and receiver can be computed as [16]:

$T L=20 \log r+\alpha r \times 10^{-3}+T L_{S R}$,

where, $r$ - transmission range $(\mathrm{m})$ and $\alpha$ - attenuation coefficient of sea water $(\mathrm{dB} / \mathrm{km})$.

Figure 5(a) represents TL due to surface reflection as a function of frequency by the curve of blue color.

\subsubsection{Surface Duct}

Sound emanating from a source in isothermal layer is prevented from spreading in all directions and is confined between the boundaries of the mixed sound channel. This propagation phenomenon is called surface duct. Transmission loss due to surface duct can be computed as follows [3]:

$$
\begin{aligned}
& T L=20 \log r+\left(\alpha+\alpha_{L}\right) r \times 10^{-3}, r<350 H^{2}(\text { short ranges }) \\
& T L=10 \log r_{o}+10 \log r+\left(\alpha+\alpha_{L}\right) r \times 10^{-3}, \quad r>350 H^{2}(\text { long ranges }) \\
& 10 \log r o=20.9+5 \log H,
\end{aligned}
$$


International Journal on Cybernetics \& Informatics (IJCI) Vol. 4, No. 2, April 2015

$\alpha_{L}=\frac{26.6 f(1.4)^{S}}{[(1452+3.5 T) H]^{1 / 2}}$

where, $\mathrm{S}=$ sea state number and $\mathrm{T}=$ temperature (degree Celsius) $\mathrm{H}=$ mixed layer depth $(\mathrm{m})$

Figure 5(a) represents TL due to surface duct as a function of frequency by the curve of green color. It can very well be inferred that transmission to medium distances is possible with moderate transmission loss.

\subsubsection{Bottom Bounce}

This is the propagation phenomenon due to sound reflection from the sea floor at grazing angles to a plain boundary between two fluids of different densities ( $\rho_{1}$ - density of sea water and $\rho_{2}-$ density of sea water with sediment $)$ and sound velocities $\left(c_{1}\right.$ - sound velocity in pure water and $c_{2}$ - sound velocity in sea bottom with sediment). The bottom bounce loss can be computed as follows [3]:

$T L_{B B}=10 \log \left[\frac{\left(m \sin \theta_{1}-\left(n^{2}-\cos ^{2} \theta_{1}\right)^{1 / 2}\right.}{\left(m \sin \theta_{1}-\left(n^{2}-\cos ^{2} \theta_{1}\right)^{1 / 2}\right.}\right]^{2}$

where, $\mathrm{m}=\frac{\rho_{2}}{\rho_{1}}$ and $\mathrm{n}=\frac{c_{1}}{c_{2}}$ and $\theta_{1}=$ Grazing angle.

The values of $\mathrm{m}$ and $\mathrm{n}$ for different sediment types in sea bottom are found in [12]

The total transmission loss as a result of bottom bounce for a near-bottom source and receiver can be computed as [3]:

$T L=20 \log r+\alpha r \times 10^{-3}+T L_{B B}$

Figure 5(b) represents TL due to bottom bounce as a function of frequency by the curve of red color. It can very well be seen that TL due to bottom bounce is much higher indicating that this is to be avoided. Hence, it is always advisable to avoid deploying the transceivers near sea floor in underwater communication networks.

\subsubsection{Convergence Zone}

Convergence zone is formed as a result of the propagation of sound from a source located near the surface. In deep water, the sound rays are bent downward as a result of the negative speed gradient forming regions of convergence zone when the upper ray of the sound beam becomes horizontal. The depth at this point is called the critical depth. The water column depth must be greater than the critical depth for the existence of convergence zones. The total transmission loss for the signal within a convergence zone is computed as follows [17]:

$T L=20 \log r+\alpha r \times 10^{-3}-C Z_{-}$gain,

where, CZ_gain refers to the convergence zone gain that can be obtained numerically [17]. The values of CZ_gain are usually found to be in the range 5dB-20 dBFigure 5(a) represents TL due 
International Journal on Cybernetics \& Informatics (IJCI) Vol. 4, No. 2, April 2015

to convergence zone as a function of frequency by the curve of magenta color.It can be inferred that transmission loss is lower within convergence zone indicating that underwater communication gets improved.

\subsubsection{Deep Sound Channel}

The deep sound channel or SOFAR (Sound Fixing and Ranging) is formed along the axis where the sound speed is minimum. The propagation to long ranges is possible if the source and receiver are located below or above near the deep sound channel axis. The total transmission loss for the deep sound channel model is computed as follows [3]:

$T L=10 \log r_{o}+10 \log r+\alpha r \times 10^{-3}$,

where, $r_{o}$ is the transition from cylindrical to spherical spreading and is calculated by:

$r_{o}=\left(\frac{r_{s}}{8}\right)\left(\frac{D_{z}}{Z_{s}}\right)^{1 / 2}$,

where, $r_{s}$ is the skip distance, $D_{s}$ is the axis depth and $z_{s}$ is the source depth.

Figure 5(a) represents TL due to deep sound channel as a function of frequency by the curve of cyan color. It can be clearly noted from the figure that transmission to long ranges are possible with low transmission loss.

\subsubsection{Reliable Acoustic Path}

When the sound propagates from a deep source to shallow receiver, transmission to moderate distances takes place through the reliable acoustic path. The path is reliable since it does not get affected by the reflection from the surface or from the ocean bottom. The transmission loss for this model can be computed as follows [18]:

$T L=20 \log r+\alpha r \times 10^{-3}$

Figure 5(a) represents TL due to reliable acoustic path as a function of frequency by the curve of black color. It can be well understood that the graph is similar to that of direct path model due to the fact that the surface and the bottom reflections do not affect the transmission signal.

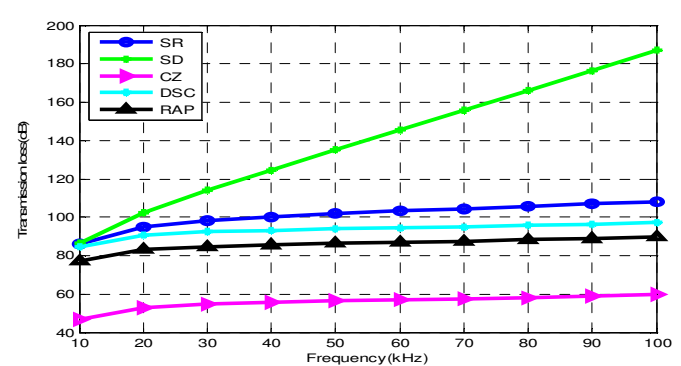

(a)

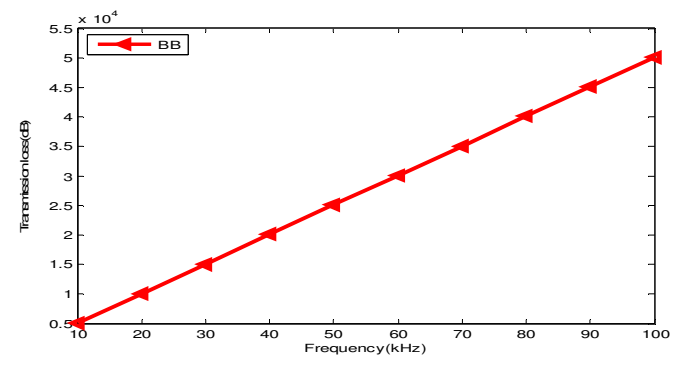

(b)

Figure 5. TL for deep water multipath models

(a) TL due to SR, SD, CZ, DSC, RAP (b) TL due to bottom bounce 
International Journal on Cybernetics \& Informatics (IJCI) Vol. 4, No. 2, April 2015

\subsection{Ambient Noise}

Ambient noise is an important underwater acoustic characteristic of the ocean. Ambient noise contains the amount of information concerned with atmosphere of the ocean, sea state of the ocean, wind speed and marine biological effects. Four basic sources model the different dominating levels of ambient noise in the ocean. They are: turbulence, waves, shipping and thermal noise. The overall power spectral density of the ambient noise is expressed in $\mathrm{dB}$ and is given by [19]:

$$
N L=\mathrm{NL}_{t b}+\mathrm{NL}_{s h}+N L_{w v}+N L_{t h}(23)
$$

where,

$$
\begin{array}{ll}
N L_{t b}=27-30 \log f, \quad f \text { in } \mathrm{KHz}, & N L_{w v}=50+7.5 w^{0.5}+20 \log f-40 \log (\mathrm{f}+0.4) \\
N L_{s h}=40+20(s-0.5)+26 \log f-60 \log (f+0.03) & N L_{t h}=-25+20 \log f \\
\text { W - windspeed }(\mathrm{m} / \mathrm{s}) \text { and } \mathrm{s}-\text { shipping activity factor }
\end{array}
$$

Figure 6 represents the total noise level and the noise levels contributed by different sources of turbulence, shipping, waves and thermal noise. It can be inferred that the turbulence noise is the dominant factor for frequencies below $20 \mathrm{~Hz}$, distant shipping is the dominant factor for frequencies in the range from $20 \mathrm{~Hz}$ to $200 \mathrm{~Hz}$, surface motion caused by wind-driven waves is the dominant factor for frequencies in the range from $200 \mathrm{~Hz}-200 \mathrm{kHz}$ and thermal noise is the dominant factor for frequencies above $200 \mathrm{kHz}$.

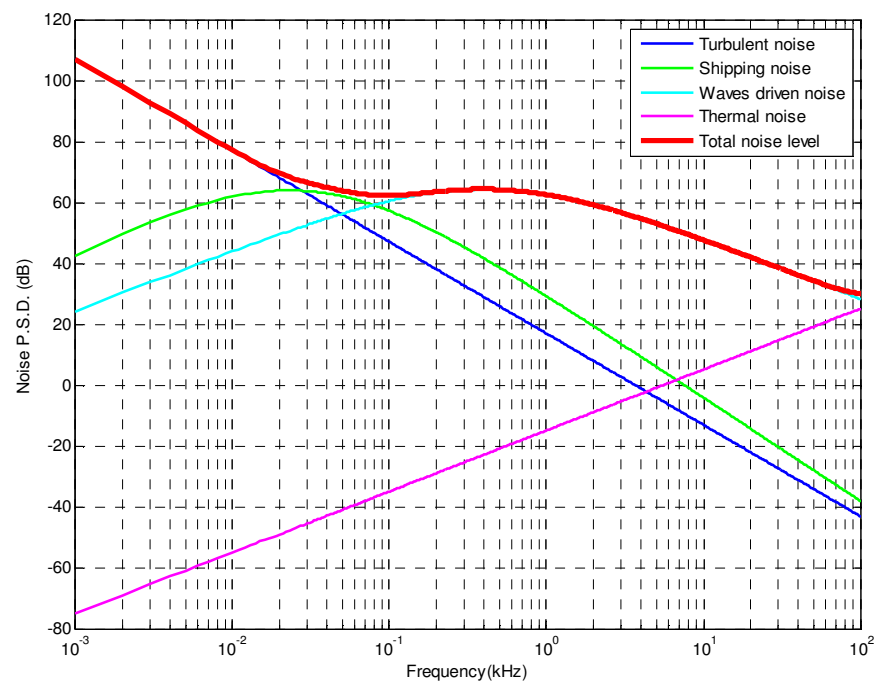

Figure 6. Ambient Noise vs Frequency 


\section{Signal to NoiSe RATiO (SNR)}

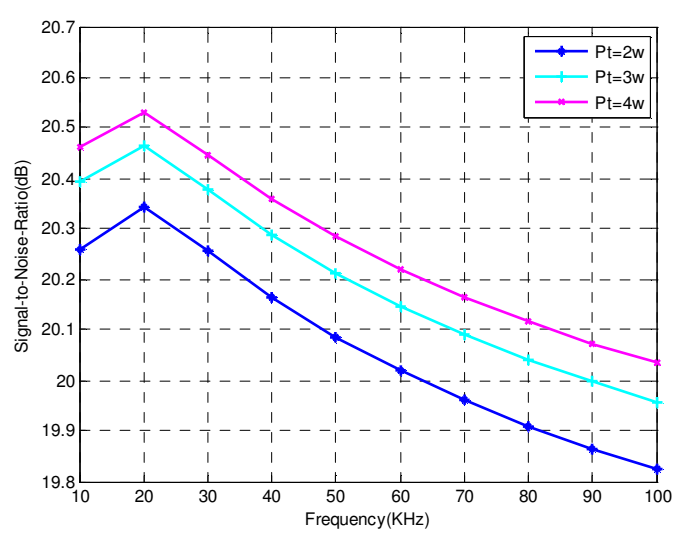

(a)

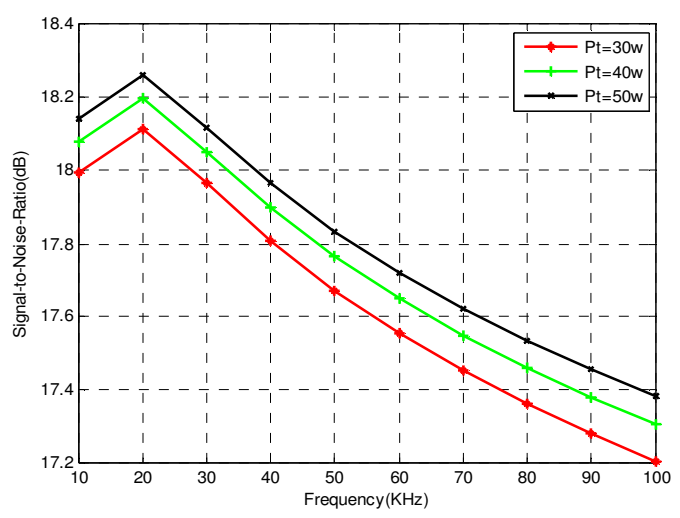

(b)

Figure 7. Signal-to-Noise Ratio as a function of frequency

(a) for shallow water model (b) for deep water model

(b)

The SNR of an underwater acoustic signal at a receiver can be computed in $\mathrm{dB}$ by the passive sonar equation [6] as follows:

$S N R=S L-T L-N L-D I$

where,

NL is the ambient noise level in ocean $(\mathrm{dB}), \mathrm{TL}$ is the transmission loss $(\mathrm{dB})$,

$\mathrm{DI}$ is the directivity index and is set to zero and SL is the source level of transmitter (dB) given by,

$S L=10 \log \left(\frac{I t}{0.67 \times 10^{(-18)}}\right)(\mathrm{dB})$, where $I_{t}$ is the transmission power intensity

In shallow water, the intensity, $I_{t}$ is given in Watts $/ \mathrm{m}^{2}$ as follows:

$I_{t}=\frac{P_{t}}{2 \times \pi \times 1 m \times z}(26)$

In deep water, $I_{t}$, is given in Watts $/ \mathrm{m}^{2}$ as follows:

$I_{t}=\frac{P_{t}}{4 \times \pi \times 1 m \times z}$, where, $P_{t}$ is the transmitter power (Watt) and $\mathrm{z}$ is the depth (m).

The relationship between SNR and frequency for shallow water are shown in Figure 7(a). SNR increases with increasing transmission power. The optimal frequency for which the maximum SNR can be achieved in shallow water is $20 \mathrm{kHz}$. The relationship between SNR and frequency for deep water are shown in Figure 7(b). SNR increases with increasing transmission power. The optimal frequency for which the maximum SNR can be achieved in deep water is $20 \mathrm{kHz}$. 


\section{International Journal on Cybernetics \& Informatics (IJCI) Vol. 4, No. 2, April 2015}

\section{CONCLUSION}

Based on the study of acoustic propagation characteristics, underwater acoustic channel models for each mode of propagation in deep water and shallow water have been presented.Our study on channel models shows that attenuation increases with frequency, long range systems have low bandwidth and short range systems have high bandwidth. The distance between transceivers and the requirements of specific applicationsdetermine the desired communication range. The ambient noise is dependent on selected frequency. Of all the six basic propagation phenomena of sound in deep water, TL is very much lower within the convergence zone providing the suggestions for the optimal deployment of source and receiver nodes in underwater communication networks. Also, SNR for both the cases are thoroughly analyzed. The optimal frequency for which maximum SNR value can be achieved is found to be $20 \mathrm{kHz}$ in shallow water and deep water as well. The numerical results obtained through analytical simulations carried out in this paper facilitate the researchers and scientists to develop higher layer communication protocols for UWCNs to guarantee for effective and reliable communication.

\section{REFERENCES}

[1] I.F.Akyildiz, D.Pompili, T.Melodia, (2005), "Underwater acoustic sensor networks: Research Challenges", AdHoc Networks Journal, (Elsevier), 3 (3).

[2] P.C. Etter, (2003), Underwater Acoustic Modeling and Simulation, 3rd ed., Spon Press, New York.

[3] R.J. Urick, (1983), Principles of Underwater Sound, 3rd ed., McGraw-Hill.

[4] E. Sozer, M. Stojanovic, J. Proakis, (1999), "Design and simulation of an underwater acoustic local area network", in: Proc. Opnetwork'99, Washington, DC, August.

[5] J.A. Catipovic, A.B. Baggeroer, K. Von Der Heydt, D. Koelsch, (1984), "Design and performance analysis of a digital telemetry system for short range underwater channel", IEEE Journal of Oceanic Engineering OE ,9 (4) 242-252.

[6] M. Chitre, S. Shahabodeen, M. Stojanovic,(2008) "Underwater acoustic communications and networking: Recent advances and future challenges", Marine Technology Society Journal, 42 (1) pp. 103-116.

[7] R. Galvin, R.F.W Coates, (1994), "Analysis of the performance of an underwater acoustic communication system and comparison with stochastic model”, IEEE Oceans'94, Brest, France, pp. III/478_III/482.

[8] X. Geng, A. Zielinski, (1995), “An eigenpath underwater acoustic communication channel model”, in: Proc. OCEANS, MTS/IEEE, Challenges Our Changing Global Environment' Conf., 2, Oct. pp. 11891196.

[9] M. Chitre, (2007), "A high-frequency warm shallow water acoustic communications channel model and measurements", Journal of the Acoustical Society America, 122 (5), pp. 2580-2586.

[10] L.M.Brekhovskikh, Y.P.Lysanov, (2003), "Fundamentals of Ocean Acoustics", 3rd edition, Springer, New York,

[11] K.V. Mackenzie, "Nine-term equation for sound speed in the oceans", Journal of acoustical Society of America 70(3)(1981) 807-812.

[12] R.E. Francois, G.R. Garrison,(1982),"Sound absorption based on ocean measurements: PartII: Boric acid contribution and equation for total absorption", Journal of the Acoustical Society of America, 72 (6), pp. 1879-1890.

[13] R.E. Francois, G.R. Garrison, (1982), "Sound absorption based on ocean measurements: Part I: Pure water and magnesium sulfate contributions", Journal of the Acoustical Society of America, 72 (3) pp. 896-907.

[14] H.W. Marsh, M.Schulkin,(1962) "Shallow water transmission", Journal of Acoustical Society of America, 34, pp. 863-864.

[15] M. Schulkin, J.A. Mercer, (1962), “Colossus revisited: A review and extension of the Marsh_Schulkin shallow water transmission loss model”,Journal of Acoustical Society of America, 34, pp. 863-864. 
International Journal on Cybernetics \& Informatics (IJCI) Vol. 4, No. 2, April 2015

[16] R. Coates ,(1998), "An empirical formula for computing the Beckhmann-Spizzichino surface reflection loss coefficient”, IEEE Transactions on Ultrosonics, Ferroelectrics, and Frequency Control, 35 (4), pp.522-523.

[17] A.W.Cox,(1974)Sonar and Underwater Sound, Lexington Books, Lexington, MA.

[18] L.E. Kinsler, A.R. Frey, A.B. Coppens, J.V. Sanders, (2000) Fundamentals of Acoustics, 4th edition, John Wiley \& Sons cop., New York.

[19] M.Stojanovic,(2006), "On the relationship between capacity and distance in an underwater acoustic channel, in: Proceedings of First ACM International Workshop on Underwater Networks (WUWNeT'06) / Mobicom, Los Angeles, CA, September.

\section{AUTHORS}

S. Anandalatchoumy receivedB.Tech. degree in 2006 and M.Tech in 2010 in Electronics and Communication Engineering from Pondicherry University, India. She has 5 years in teaching experience. She is currently working as Senior Assistant Professor in the Department of Electronics and Communication Engineering, Christ college of Engineering and Technology, Pondicherry, India. She is currently pursuing her Ph.D. degree in Pondicherry Engineering College. Her areas of interest are Wireless communication and Sensor Networks.

G. Sivaradje received B.E degree from University of Madras in 1991 and M.Tech. in 1996 in Electronics and Communication Engineering and $\mathrm{Ph}$. D degree from Pondicherry University, India in 2006. He has 16 years in teaching and 4 years of industrial experience. $\mathrm{He}$ is currently working as Professor in the Department of Electronics and Communication Engineering, Pondicherry Engineering College, Pondicherry, India. He has 16 Publications in reputed International and National Journals and published research papers more than 50 in International and National Conferences. His areas of interest are Wireless Networking and

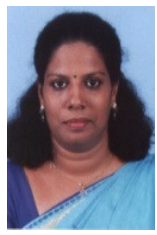
Image Processing. 